\title{
An empirical comparison of atypical bulimia nervosa and binge eating disorder
}

L.F. Fontenelle $e^{1,2}$ M.V. Mendlowicz ${ }^{3}$, R.O. Moreira ${ }^{1}$ and J.C. Appolinario ${ }^{1}$

\author{
${ }^{1}$ Grupo de Obesidade e Transtornos Alimentares, Instituto Estadual de Diabetes e \\ Endocrinologia do Rio de Janeiro, e Instituto de Psiquiatria, \\ Universidade Federal do Rio de Janeiro, Rio de Janeiro, RJ, Brasil \\ 2Programa de Ansiedade e Depressão, 3Programa de Ética e Psiquiatria Forense, \\ Instituto de Psiquiatria, Universidade Federal do Rio de Janeiro, Rio de Janeiro, \\ RJ, Brasil
}

\section{Correspondence \\ L.F. Fontenelle \\ Rua Lopes Trovão, 88 \\ Apto. 1501, Bloco A \\ 24220-071 Niterói, RJ \\ Brasil \\ Fax: +55-21-2239-4919 \\ E-mail: Ifontenelle@gmail.com \\ Research supported by CNPq (No. 141517/00-0).}

Received November 26, 2004 Accepted May 20, 2005

\begin{abstract}
The International Classification of Diseases, 10th edition (ICD-10) defines atypical bulimia nervosa $(\mathrm{ABN})$ as an eating disorder that encompasses several different syndromes, including the DSM-IV binge eating disorder (BED). We investigated whether patients with $B E D$ can be differentiated clinically from patients with $A B N$ who do not meet criteria for BED. Fifty-three obese patients were examined using the Structured Clinical Interview for DSM-IV and the ICD-10 criteria for eating disorders. All volunteers completed the Binge Eating Scale (BES), the Beck Depression Inventory, and the Symptom Checklist-90 (SCL-90). Individuals fulfilling criteria for both ABN and $\operatorname{BED}(\mathrm{N}=18), \mathrm{ABN}$ without $\mathrm{BED}(\mathrm{N}=16)$, and obese controls $(\mathrm{N}$ $=19)$ were compared and contrasted. Patients with $A B N$ and BED and patients with $\mathrm{ABN}$ without $\mathrm{BED}$ displayed similar levels of binge eating severity according to the BES $(31.05 \pm 7.7$ and $30.05 \pm 5.5$, respectively), which were significantly higher than those found in the obese controls $(18.32 \pm 8.7 ; \mathrm{P}<0.001$ and $\mathrm{P}<0.001$, respectively). When compared to patients with $\mathrm{ABN}$ and $\mathrm{BED}$, patients with $\mathrm{ABN}$ without BED showed increased lifetime rates of agoraphobia $(\mathrm{P}=$ $0.02)$ and increased scores in the somatization $(1.97 \pm 0.85$ vs $1.02 \pm$ $0.68 ; \mathrm{P}=0.001)$, obsessive-compulsive $(2.10 \pm 1.03$ vs $1.22 \pm 0.88 ; \mathrm{P}$ $=0.01)$, anxiety $(1.70 \pm 0.82$ vs $1.02 \pm 0.72 ; \mathrm{P}=0.02)$, anger $(1.41 \pm$ 1.03 vs $0.59 \pm 0.54 ; \mathrm{P}=0.005)$ and psychoticism $(1.49 \pm 0.93$ vs 0.75 $\pm 0.55 ; \mathrm{P}=0.01$ ) dimensions of the SCL- 90 . The BED construct may represent a subgroup of $\mathrm{ABN}$ with less comorbities and associated symptoms.
\end{abstract}

Despite some subtle differences in the way the International Classification of Diseases, 10th edition (ICD-10) and the Diagnostic and Statistical Manual of Mental Disorders, 4th edition (DSM-IV) describe bulimia nervosa (e.g., the binge eating-related
Key words

- Atypical bulimia nervosa

- Binge eating disorder

- Anxiety

- Obesity loss of control, the frequency of compensatory behaviors, and the exclusion criteria for bulimia nervosa are not mentioned in ICD10), it is clear that their diagnostic criteria largely overlap. More striking differences are seen, however, in the way these manuals 
conceptualize atypical forms of eating disorders characterized by binge eating, namely the DSM-IV binge eating disorder (BED) and the ICD-10 atypical bulimia nervosa (ABN).

BED is a newly recognized diagnostic entity characterized by episodic uncontrolled consumption of large amounts of food (binge eating) in the absence of the inappropriate compensatory methods that characterize bulimia nervosa, including vomiting, laxative abuse, fasting, or excessive exercise following a binge eating (1). The provisional criteria for BED, which have been included in DSM-IV, Appendix B, state that the individual must experience significant distress related to binge eating and must present several behavioral indicators of loss of control (2). It has been demonstrated that BED is associated with higher rates of axis I and axis II psychiatric disorders $(3,4)$.

$\mathrm{ABN}$ is characterized by the lack of one or more of the key features listed for bulimia nervosa in patients with an otherwise fairly typical clinical picture (including or not compensatory methods) (5). According to the ICD-10, this most commonly applies to people with normal or even excessive weight but with typical periods of over-eating followed by vomiting or purging (5). Although described almost 10 years ago, there is an almost absolute dearth of empirical studies investigating the nosological status of this condition. Based on the official definitions, one might suppose that the ICD-10 ABN is a broader concept than the DSM-IV BED and that the former may encompass the latter.

In the present study with female obese individuals, we compared the psychiatric status of patients with $\mathrm{ABN}$ who do not fulfill the criteria for $\mathrm{BED}(\mathrm{ABN} \neq \mathrm{BED})$, patients with $A B N$ who do fulfill the criteria for $\mathrm{BED}(\mathrm{ABN}=\mathrm{BED})$ and patients without any of these diagnoses. We hypothesized that patients with $\mathrm{ABN}(\mathrm{ABN} \neq \mathrm{BED})$ would present a more severe psychiatric profile as determined with diagnostic instruments and psychopathological rating scales.
Obese individuals recruited through advertisements in newspapers and referrals from clinicians were consecutively interviewed by the research staff of the Obesity and Eating Disorders Group of the State Institute of Diabetes and Endocrinology of Rio de Janeiro. They were invited to join a weight loss program if they were 18 to 65 years old and had a body mass index $\geq 30 \mathrm{~kg} /$ $\mathrm{m}^{2}$ and $<45 \mathrm{~kg} / \mathrm{m}^{2}$. Exclusion criteria were: significant medical, neurological and endocrine disorders, inability to read and fill out forms and questionnaires, psychosis, and severe personality disorder (i.e., marked antisocial, borderline, and histrionic features that might compromise the participation in the weigh loss program).

The protocol was approved by the Ethics Committee of the Institution.

After signing an informed consent form, patients were examined by an experienced research psychiatrist (LFF) using the Structured Clinical Interview for DSM-IV (6). On that occasion, the interviewer was unaware of the hypothesis of the study. All individuals completed Portuguese versions of the Binge Eating Scale (7), the Symptom Checklist-90 (SCL-90) (Versiani M, unpublished results), and the Beck Depression Inventory (8). Most patients were evaluated during the period between January and March, 2001.

For the purpose of this study, a convenient sample of 53 patients had their charts reviewed. Eighteen patients meeting the DSM-IV criteria for current BED were identified; since the diagnostic criteria for BED and $\mathrm{ABN}$ overlapped in these cases (i.e., the patients with BED always fulfilled the diagnosis for $\mathrm{ABN}$ too), this group was named $\mathrm{ABN}=\mathrm{BED}$. The 35 patients who did not fulfill the DSM-IV criteria for current BED had their medical records scrutinized by means of a checklist developed by us that included the ICD-10 criteria for ABN. This procedure led to the identification of an additional 16 individuals who met criteria solely for $\mathrm{ABN}$. This latter group was named $\mathrm{ABN}$ 
$\neq \mathrm{BED}$ to emphasize the fact that their ABN did not correspond to a simultaneous diagnosis of BED. Finally, the last 19 individuals who did not satisfy diagnostic criteria for $\mathrm{BED}$ or for $\mathrm{ABN}$ were classified as obese controls. The three groups were compared for lifetime diagnosis of psychiatric comorbidity using the ANOVA (with post hoc Tukey's test) and chi-square tests, with the level of significance set at $5 \%$.

Individuals from the $\mathrm{ABN}=\mathrm{BED}$ and $\mathrm{ABN} \neq \mathrm{BED}$ groups displayed comparable lifetime prevalence rates of major depressive disorder and similar levels of binge eating severity (as assessed on the basis of the Binge Eating Scale scores), both of which were significantly higher than those found in obese controls $(\mathrm{P}<0.03$ and $\mathrm{P}<0.001$, respectively, for $\mathrm{ABN}=\mathrm{BED}$; and $\mathrm{P}<0.02$ and $\mathrm{P}<0.001$, respectively, for $\mathrm{ABN} \neq$ $\mathrm{BED})$. When compared to $\mathrm{ABN}=\mathrm{BED}$, $\mathrm{ABN} \neq \mathrm{BED}$ showed higher lifetime rates of agoraphobia $(\mathrm{P}=0.02)$ and more elevated scores in the somatization $(\mathrm{P}=0.001)$, obsessive-compulsive $(\mathrm{P}=0.01)$, anxiety $(\mathrm{P}=$ $0.02)$, anger $(\mathrm{P}=0.005)$, and psychoticism $(\mathrm{P}=0.01)$ SCL-90 dimensions (Table 1).

The diagnosis of ABN may be exces-

\begin{tabular}{|c|c|c|c|c|}
\hline & $\begin{array}{c}A B N=B E D \\
(N=16)\end{array}$ & $\begin{array}{c}A B N=B E D \\
(N=18)\end{array}$ & $\begin{array}{l}\text { Control group } \\
\qquad(N=19)\end{array}$ & $\mathrm{P}$ \\
\hline Age & $37.45(6.55)$ & $36.11(10.64)$ & $32.74(9.38)$ & 0.29 \\
\hline BMI & $38.11(5.84)$ & 36.91 (3.31) & $36.55(3.53)$ & 0.54 \\
\hline BES & $31.05(7.71)^{\star}$ & $30.05(5.51)^{*}$ & $18.32(8.72)$ & 0.000 \\
\hline BDI & $25.43(9.96)^{*}$ & $20.44(11.06)$ & $13.05(7.31)$ & 0.001 \\
\hline Any mood disorder & $8^{*}$ & $10^{*}$ & 3 & 0.03 \\
\hline Major depressive episode & $7^{*}$ & $8^{*}$ & 2 & 0.03 \\
\hline Bipolar disorder & 1 & 2 & 0 & 0.21 \\
\hline Dysthymic disorder & 0 & 0 & 2 & 0.12 \\
\hline Any anxiety disorder & $9^{*}$ & 8 & 3 & 0.009 \\
\hline Panic disorder & 0 & 0 & 0 & - \\
\hline Agoraphobia & $4^{*+}$ & 0 & 0 & 0.003 \\
\hline Social phobia & $5^{*}$ & 2 & 1 & 0.05 \\
\hline Specific phobia & 4 & $6^{*}$ & 1 & 0.04 \\
\hline Obsessive-compulsive disorder & 2 & 1 & 1 & 0.56 \\
\hline Post-traumatic stress disorder & 0 & 0 & 0 & - \\
\hline Generalized anxiety disorder & $5^{*}$ & 1 & 1 & 0.02 \\
\hline \multicolumn{5}{|l|}{ SCL-90 } \\
\hline Somatization & $1.97(0.85)^{\star+}$ & $1.02(0.68)$ & $0.91(0.55)$ & 0.000 \\
\hline Obsessive-compulsive & $2.10(1.03)^{\star+}$ & $1.22(0.88)$ & $0.93(0.66)$ & 0.001 \\
\hline Interpersonal sensitivity & $1.87(0.96)$ & $1.22(0.78)$ & $1.14(0.81)$ & 0.04 \\
\hline Depression & $2.09(1.13)^{\star}$ & $1.46(0.84)$ & $1.08(0.74)$ & 0.009 \\
\hline Anxiety & $1.70(0.82)^{\star+}$ & $1.02(0.72)$ & $0.84(0.59)$ & 0.003 \\
\hline Anger-hostility & $1.41(1.03)^{\star+}$ & $0.59(0.54)$ & $0.57(0.48)$ & 0.002 \\
\hline Phobic anxiety & $1.20(1.02)$ & $0.58(0.62)$ & $0.63(0.56)$ & 0.04 \\
\hline Paranoid ideation & $1.58(1.03)^{\star}$ & $0.98(0.60)$ & $0.91(0.71)$ & 0.03 \\
\hline Psychoticism & $1.49(0.93)^{*+}$ & $0.75(0.55)$ & $0.64(0.58)$ & 0.002 \\
\hline
\end{tabular}

Data are reported as mean with $S D$ given in parentheses. $A B N=$ atypical bulimia nervosa; $B E D=$ binge eating disorder; $\mathrm{BMI}=$ body mass index; $\mathrm{BES}=$ Binge Eating Scale; $\mathrm{BDI}=$ Beck Depression Inventory; SCL-90 = Symptom Checklist-90.

${ }^{*} \mathrm{P}<0.05$ compared to the control group. ${ }^{+} \mathrm{P}<0.05$ compared to the $A B N=B E D$ group. $P$ values in the last column are comparisons between the three groups. ANOVA (with post hoc Tukey's test) and chi-square tests were employed for continuous and categorical variables, respectively. 
sively broad, encompassing individuals with different types and degrees of eating psychopathology. In our study, $\mathrm{ABN}=\mathrm{BED}$ and $\mathrm{ABN} \neq \mathrm{BED}$ displayed similar lifetime prevalence rates of major depressive disorder and severity of binge eating, which were nevertheless significantly higher than those found in obese controls. However, when compared to $\mathrm{ABN}=\mathrm{BED}, \mathrm{ABN} \neq \mathrm{BED}$ showed more elevated lifetime rates of agoraphobia and higher scores in the somatization, obsessive-compulsive, anxiety, angerhostility, and psychoticism dimensions of the SCL-90.

We agree with the assumption of Tobin et al. (9) that, although much research and theoretical work has been focused on binge eating as the core psychopathologic feature of bulimia nervosa and BED, compensation may be an even stronger predictor of comorbid psychopathology. Tobin et al. (9) compared patients with bulimia nervosa with and without purging to patients with BED and to patients who compensate but do not binge or meet weight criteria for anorexia (a condition called compensatory eating disorder). There were no significant differences between the patients with bulimia nervosa (with or without purging) and the patients with compensatory eating disorder in the SCL-90-R and on the Eating Disorders Inventory scores. However, all three groups showed more comorbid disorders than the patients with BED. In fact, several older studies comparing individuals with bulimia nervosa and BED have found that the former reported more comorbid psychopathology $(10,11)$, disturbed attitudes toward weight and shape, eating pathology, depressive symptoms $(11,12)$, dietary restraint (13), and ineffectiveness (14). The frontiers of BED with non-purging forms of bulimia nervosa, however, remains a matter of debate $(15,16)$.

We are not aware of any previous studies assessing the nosological status of $\mathrm{ABN}$ and comparing it to BED and to obese controls. However, at least one study has contrasted groups of individuals who might superficially resemble our patients, with negative results. Peterson (17) failed to show any difference between individuals with BED with and without a history of purging behaviors in terms of comorbid psychopathology, dietary restraint, severity of eating pathology, and attitudinal disturbance in self-esteem and body image. One possible explanation for the differences between this study and our own findings is that Peterson (17) excluded patients with a recent history of purging behaviors (i.e., in the previous 6 months), while, in contrast, some of our volunteers were currently exhibiting such compensatory maneuvers.

It is conceivable that, with one exception (i.e., the SCL-90 psychoticism subscale), the features that were found to differentiate ABN from BED (the somatization, obsessive-compulsive, anxiety, and anger-hostility symptoms subscales of the SCL-90, and the comorbid diagnosis of agoraphobia) pertain to a hypothetical spectrum of anxiety symptoms. It remains to be established, however, whether anxiety symptoms predispose to or result from compensatory behaviors in ABN. Lynch et al. (18), for example, have found that students reporting binge eating and compensatory activities reported greater negative affect preceding binge episodes than those who did not compensate. In addition, contrary to expectations, negative affect did not diminish, but rather increased significantly following binge episodes, and decreased immediately before and after compensatory activities.

Another interesting aspect related to the way $\mathrm{ABN}$ differed from $\mathrm{BED}$ is that the former exhibited more severe symptoms in the psychoticism dimension of SCL-90. In fact, one of the symptoms that contribute to the final score in this dimension (i.e., "The idea that something serious is wrong with your body") may clearly reflect body image dissatisfaction, which is one of the diagnostic criteria of $\mathrm{ABN}$ in the ICD-10 (“...There 
is self-perception of being too fat, with an intrusive dread of fatness (usually leading to underweight)..."), but not of BED in the DSM-IV. Thus, the relationship between ABN and psychoticism in SCL-90 may be somewhat circular, rather than reflecting a true psychiatric comorbidity.

Our study has some significant limitations. Firstly, it included a relatively small number of patients, 53. Secondly, it was a chart review. Finally, we have employed a checklist with the ICD-10 diagnostic criteria for $A B N$ to identify such patients. It would be interesting to test our findings in a larger number of participants, examined prospectively with validated instruments specially designed to identify ICD-10 and DSM-IV eating disorders simultaneously, such as, for example, the Structured Interview for Anorexic and Bulimic Disorders for DSM-IV and ICD-10 (19).

We believe that, by allowing the inclusion of patients with and without compensatory behaviors, the ICD-10 diagnostic criteria for $\mathrm{ABN}$ may encompass patients suffering from different levels of the eating disorder and comorbid psychopathology. In addition, rather than being clearly "atypical", the diagnosis of ABN may include individuals with a fairly typical bulimia nervosa (i.e., the $\mathrm{ABN} \neq \mathrm{BED}$ group), suffering from significantly higher levels of psychopathology.

\section{References}

1. Devlin MJ (1996). Assessment and treatment of binge-eating disorder. Psychiatric Clinics of North America, 19: 761-772.

2. American Psychiatric Association (1994). Diagnostic and Statistical Manual of Mental Disorders. 4th edn. American Psychiatric Press, Washington, DC, USA.

3. Yanovski SZ, Nelson JE, Dubbert BK et al. (1993). Association of binge eating disorder and psychiatric comorbidity in obese subjects. American Journal of Psychiatry, 150: 1472-1479.

4. Fontenelle LF, Vltor Mendlowicz M, de Menezes GB et al. (2003). Psychiatric comorbidity in a Brazilian sample of patients with bingeeating disorder. Psychiatry Research, 119: 189-194.

5. World Health Organization (1993). The ICD-10 Classification of Mental and Behavioral Disorders. Clinical Descriptions and Diagnostic Guidelines. World Health Organization, Geneva, Switzerland.

6. First MB, Spitzer RL, Gibbom M et al. (1996). Structured Clinical Interview for DSM-IV Axis I Disorders: Patients Edition (SCID-I/P, Version 2.0). Biometrics Research Department, New York State Psychiatric Institute, New York.

7. Appolinario JC, Freitas SR, De Menezes GB et al. (2001). Validation of the Portuguese Version of the Binge Eating Scale. Presented at the Annual Meeting of the American Psychiatric Association, New Orleans, LO, USA, May 5-10 (Abstract).

8. Gorenstein C, Andrade L, Vieira Filho AH et al. (1999). Psychometric properties of the Portuguese version of the Beck Depression Inventory on Brazilian college students. Journal of Clinical Psychology, 55: 553-562.

9. Tobin DL, Griffing A \& Griffing S (1997). An examination of subtype criteria for bulimia nervosa. International Journal of Eating Disorders, 22: 179-186.

10. Kirkley BG, Kolotkin RL, Hernandez JT et al. (1992). A comparison of binge-purgers, obese binge eaters and obese nonbinge eaters on the MMPI. International Journal of Eating Disorders, 12: 221-228.

11. Raymond NC, Mussell MP, Mitchell et al. (1995). An age-matched comparison of subjects with binge eating disorder and bulimia nervosa. International Journal of Eating Disorders, 18: 135-143.

12. Crow SJ, Zander KM, Crosby RD et al. (1996). Discriminant function analysis of depressive symptoms in binge eating disorder, bulimia nervosa, and major depression. International Journal of Eating Disorders, 19: 399-404.

13. Marcus MD, Smith D, Santelli $R$ et al. (1992). Characterization of eating disordered behavior in obese binge eaters. International Journal of Eating Disorders, 12: 249-255.

14. Fichter MM, Quadflieg N \& Brandl B (1993). Recurrent overeating: an empirical comparison of binge eating disorder, bulimia nervosa, and obesity. International Journal of Eating Disorders, 14: 1-16.

15. Cooper $Z$ \& Fairburn CG (2003). Refining the definition of binge eating disorder and nonpurging bulimia nervosa. International Journal of Eating Disorders, 34 (Suppl): S89-S95.

16. Striegel-Moore RH, Cachelin FM, Dohm FA et al. (2001). Comparison of binge eating disorder and bulimia nervosa in a community sample. International Journal of Eating Disorders, 29: 157-165.

17. Peterson CB (1998). Binge eating disorder with and without a history of purging symptoms. International Journal of Eating Disorders, 24: 251-257.

18. Lynch WC, Everingham A, Dubitzky $J$ et al. (2000). Does binge eating play a role in the self-regulation of moods? Integrative Physiological and Behavioral Science, 35: 298-313.

19. Fichter MM, Herpertz S, Quadflieg N et al. (1998). Structured Interview for Anorexic and Bulimic Disorders for DSM-IV and ICD-10: updated (third) revision. International Journal of Eating Disorders, 24: $227-249$. 